\title{
Laboratory investigation of fungal infections of the central nervous system
}

\author{
John A. Davis, Daniel J. Costello*, Nagagopal Venna* \\ Division of Infectious Diseases and *Department of Neurology, Massachusetts General Hospital, USA
}

\begin{abstract}
While fungal infections of the central nervous system (CNS) are relatively rare, fungal pathogens are increasingly being recognized as an important etiology of CNS infections, particularly amongst the growing immunocompromized population. In this paper we aim to provide a practical approach to the diagnosis of fungal infections of the CNS, review some of the diagnostic methods currently available and discuss diagnosis of certain pathogens of particular interest to the practicing neurologist.
\end{abstract}

Key words: Fungal encephalitis, fungal infection, fungal meningitis

While the majority of infections in the central nervous system (CNS) are caused by bacteria and viruses, fungi are increasingly being recognized as important pathogens, particularly in immunocompromized patients. New fungal pathogens are being discovered and new syndromes are being described for both known and emerging fungal pathogens. The elucidation of the spectrum of disease associated with fungal infections of the CNS is necessarily dependent upon the advancement of diagnostic methods in order to establish etiology. This paper is intended to review the current literature regarding the laboratory investigation of fungal infections of the CNS.

Fungal infections of the CNS may be categorized in a variety of ways. In this paper, we will first consider the diagnostic approach to discrete presenting clinical syndromes. Then, consideration will be given to specific diagnostic methods. Finally, the paper will turn to a more detailed discussion of laboratory investigation of several key pathogens of interest in clinical practice.

\section{Clinical Syndromes}

As with other infectious illnesses of the CNS, fungal infections of the CNS may present clinically in many ways. Some of these are considered below.

\section{Meningitis/encephalitis}

This heading subsumes presentations ranging from subacute and chronic meningitis syndromes, to meningoencephalitis syndromes. Meningitis may result in subacute hydrocephalus and lead to presentation with a "normal-pressure" hydrocephalus or obstructive hydrocephalus (due to arachnoid villus scarring). These are most often caused by chronic infections with yeasts or the dimorphic fungi. The diagnostic approach typically involves brain magnetic resonance imaging (MRI) or computed tomography (CT) and cerebrospinal fluid (CSF) sampling as initial investigations.

\section{Meningovascular syndromes}

Subacute meningitis due to several of the true molds (particularly Aspergillus species) may lead to an angioinvasive disease involving the arterial trunks near the CSF cisterns at the base of the brain. The resultant arteritis may lead to infarction of the brain tissue, particularly in a vascular territory. Likewise, invasive fungal infections of the base of the skull (as seen with the Mucorales in the orbits or sinuses) may involve adjoining distal parts of the carotid arteries and their branches. In general, these two infectious syndromes present as complicated strokes, accompanied by symptoms and signs of meningitis. ${ }^{[1-3]}$ Examination of the CSF (in the case of the former) or of infected tissue (in the case of the latter) is the key to diagnosis of these disorders.

\section{Focal infection/mass lesions}

Patients with these infections usually present with focal neurological symptoms or seizures, often in conjunction with headache. Patients will often exhibit focal deficits on clinical examination, prompting imaging of the brain by CT or MRI. Such imaging 
usually reveals a discrete mass lesion, with or without accompanying contrast enhancement and occasionally with displacement of adjacent structures indicative of mass effect. Occasionally, such a lesion will be found upon routine imaging in patients with only minimal or nonspecific symptoms. Such lesions tend to be smaller and are more often located in non-eloquent neuroanatomical sites. The diagnostic approach has typically already included imaging. In such settings, CSF examination by lumbar puncture (LP) is of limited value and may risk brain herniation and thus may be contraindicated. This is especially true if the lesions are in the posterior fossa causing effacement or displacement of the fourth ventricle or incipient displacement of the cerebellar tonsil into the foramen magnum or less commonly, upward herniation of the cerebellar vermis through the tentorial notch. Neuroradiographic evidence of impending uncal herniation, displacement of the upper brainstem or lateral displacement of the midline structures (even in the supratentorial compartment) poses a significant risk of provoking neurological deterioration after LP. Therefore, the diagnostic approach in such a presentation may necessitate invasive techniques such as stereotactic biopsy or guided aspiration.

\section{Spinal cord syndromes}

These clinical syndromes vary in presentation based on the precise level(s) at which the cord is affected and the extent of the involvement of the cord. Infectious processes giving rise to spinal cord syndromes range from epidural abscess to focal spinal meningitis to frank fungal myelitis. As with the prior two broad classes of clinical syndromes, the diagnostic approach to focal lesions most often involves guided sampling of tissue/abscess material, whereas the diagnostic approach to a more diffuse meningitis or myelitis involves CSF sampling (ideally focal) and occasionally, tissue sampling (such as meningeal biopsy or cord biopsy).

\section{Foreign body-associated fungal infections}

These are syndromes most commonly encountered in the setting of a ventricular shunt (peritoneal or pleural) or a ventricular or lumbar drain, though in theory any foreign body that is contiguous with the meninges, the brain tissue or in communication with the CSF can lead to a fungal infection. Infections associated with CSF shunts/drains can be difficult to diagnose, due not only to their broad spectrum of presentation, but also due to the localized nature of the infection. For example, it is not uncommon for an infection along one portion of a shunt to give rise primarily to a localized infectious response, which may not be manifest in CSF sampled from a distant site. In addition, infections along some aspects of indwelling devices may be relatively sequestered from host immune responses, meaning that they will generate minimal, if any, signs, symptoms or classical laboratory findings associated with other inflammatory responses to CNS infections.

\section{Skull-base infections}

These syndromes include infections such as rhinocerebral mucormycosis, fungal osteomyelitis and fungal sinusitis. These infections can often extend to the CNS, occasionally with devastating consequences. The diagnostic approach to these infections is primarily that taken for each of the individual entities as they present without CNS involvement. These have been reviewed elsewhere in great detail and are not presented here. ${ }^{[4-6]}$

\section{Peripheral nervous system syndromes}

While the purpose of this paper is to review the diagnostic methods appropriate for CNS fungal infections, it is important to remember, from a clinical presentation standpoint, that fungal infections of the CNS may manifest themselves with concomitant or even primary peripheral nerve findings such as radiculopathy, plexopathy and neuropathy. While many such presentations have been reported in the literature, more common presentations include multiple cranial palsies and lumbosacral nerve root involvement with cauda equina syndrome.

\section{Diagnostic Investigations}

When a clinician suspects or wishes to investigate the possibility of fungal infection, there are a number of clinical and laboratory practice points that merit mention.

\section{Clinical considerations}

While the mainstay of diagnosis of fungal CNS infections rests with the laboratory, there are many important clinical clues to the presence of a fungal infection. These may come from the history of the clinical syndrome itself, including host factors [Table 1] or from clinical examination findings. Overall it should be noted that the incidence of fungal infections of the CNS, as opposed to the other etiologies of CNS infections, is rather low. Therefore, tests for

Table 1: Host factors that predispose to fungal infection

Human immunodeficiency virus/acquired immunodeficiency syndrome

(Cortico) steroid use

Neutropenia, especially prolonged

White blood cell defects

Acidosis

Diabetes mellitus

Intravenous drug use

Violation of the blood brain barrier (trauma, surgery, etc.)

Lymphoreticular malignancy

Iron chelation therapy 
fungal infections should be ordered less as a routine surveillance measure than as a specific test to explore a clinical concern or relevant differential diagnosis. This is particularly important when considering that CSF is a relatively precious commodity and the amount needed for any diagnostic test may necessarily exclude the performance of another test. As with CSF testing for many other infectious pathogens, the larger the volume of CSF allotted to fungal testing (particularly culture and staining), the greater the diagnostic yield. Conversely, small sample volumes (sometimes <1cc) encountered with an "add-on" fungal test may not be of sufficient size to permit diagnosis, even in the case of an otherwise significant organism burden. In addition, many fungal examinations require a significant investment of time. It is crucial to coordinate sample collection and processing with appropriate laboratory personnel to ensure proper triage and handling of specimens of interest. Such logistics should be planned well in advance of the time of specimen collection, in order to maximize diagnostic yield and minimize what might otherwise be an injudicious collection and use of samples. ${ }^{[7-9]}$

\section{CSF examination}

\section{Cell counts/differential}

The classical finding for fungal meningitis is that of a lymphocytic or monocytic pleocytosis. Notable exceptions include the true molds (such as Aspergillus spp.) and Blastomyces, which typically cause a neutrophilic pleocytosis. The presence of eosinophils, particularly an eosinophilic predominance, should raise suspicion of Coccidioides immitis as pathogen, though CSF eosinophilia has rarely been reported with other fungal pathogens such as Cryptococcus neoformans. ${ }^{[10,11]}$ Cell counts of CSF in the settings of fungal infection of foreign bodies and fungal spaceoccupying lesions depend on the extent to which such lesions abut the meninges (and therefore affect the CSF). Those that are close to the meninges may engender the typical parameningeal formula, whereas intra-parenchymal lesions may be associated with no CSF abnormalities.

\section{Chemistries (TP/Glucose)}

The typical finding for fungal meningitis is hypoglycorrachia: lowered CSF glucose level, typically defined as a glucose level less than one half of that seen in a simultaneous plasma sample, when taken at steady state. Such hypoglycorrhachia is usually seen in combination with an elevated CSF total protein level. Protein levels in excess of 1g/dL (Froin's syndrome) tend to occur in AIDS-associated Cryptococcus neoformans meningitis and usually imply a subarachnoid block to the circulation of CSF. While elevated CSF protein and low CSF glucose are common with fungal meningitis, it should be noted that these findings are by no means specific for fungal causes of meningitis. The list of causes of low CSF glucose in fact includes many noninfectious etiologies as well. In addition, as with cell counts as noted above, such findings may be absent in the setting of fungal abscess or foreign body-associated fungal infection.

\section{Gram's stain/fungal stain}

The Gram's stain is notoriously insensitive in most cases of fungal meningitis, save for Cryptococcus neoformans meningitis in AIDS patients. In the latter setting, a relatively high organism burden leads to a much higher concentration of yeast in the CSF, making detection on Gram's stain alone feasible. Likewise, the high organism burden encountered in the setting of AIDS-associated Cryptococcal meningitis has made possible the use of the India ink preparation (a black carbon pigment solution). The polysaccharide capsule of the Cryptococcal organisms gives rise to the classic "halo sign" when viewed against a background of India ink [Figure 1]. Larger volumes of CSF and the use of concentrating procedures (involving centrifugation of CSF samples), have increased the sensitivity of staining methods for detection of pathogens, including fungal organisms. Many modern laboratories rely instead on fluorescent assays (such as Calcofluor white) for the detection of fungal organisms specifically. These assays require access to a fluorescence microscope. While these methods do not increase concentration of fungal organisms, they do increase the signal-to-noise ratio over most other stains, leading to an increase in the detection of present fungal pathogens. Staining methods for CSF are less helpful in the setting of fungal foreign body infection and generally unhelpful for the identification of a fungal pathogen in the setting of a focal CNS lesion. However, fungal stains of an abscess aspirate or of a tissue specimen, are diagnostic. While staining can be helpful, particularly when culture samples are compromised (e.g., when a patient has been on antifungal therapy prior to sample procurement), culture is the preferred method of identification.

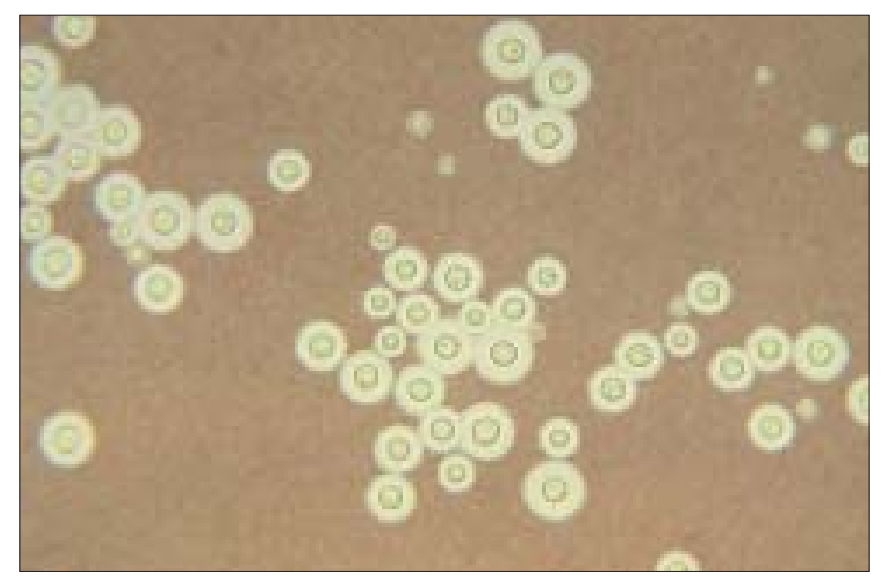

Figure 1: Cryptococcus neoformans on an India ink stain. Source: CDC/Dr. Leanor Haley, public domain 


\section{Culture}

As with Gram's stain, when compared to the use of culture for bacterial causes of meningitis, culture data of the CSF in fungal causes of meningitis are far less sensitive. However, as with staining techniques, methods of concentration have increased the culture yield. Therefore, despite a relatively low organism burden, with a high enough starting volume of CSF, a reasonable sensitivity of fungal culture can be obtained. Depending on the clinical scenario, cumulative volumes of greater than 50cc of CSF have been required in order to make a diagnosis by culture. It should be noted that targeted CSF sampling might have some value. It has been observed that CSF sampling from a lumbar tap may have a lower diagnostic yield than CSF obtained from a cisternal or C1-C2 tap in the setting of fungal meningitis. While these observations have not been subjected to validation in a randomized, controlled trial, such measures should be considered when repeated attempts at LP (and other noninvasive measures) have failed to yield a diagnosis and when fungal meningitis remains on the differential. Again, as with staining techniques, culture of CSF in the setting of focal lesions or foreign body-associated infections is singularly unhelpful. The preferred method of diagnosis in these situations is culture of abscess aspirate, culture of tissue sample(s) or culture of (part of the) removed foreign body. Overall, culture data are superior to staining data as they allow not only definitive identification of an organism (whereas staining may only narrow the differential of possible fungal organisms), but culture also allows for the determination of antifungal agent susceptibility testing, thereby permitting targeted therapeutic decisions. ${ }^{[12]}$

\section{Fungal antigen detection}

In situations when organism burden is expected to be too low to reasonably yield a positive culture, antigen detection methods may prove useful. Of note, these methods do not distinguish between viable and nonviable organisms and thus should not be relied upon for test of cure. There are two primary means of antigen detection. The first involves antigen/antibody complex formation (with externally supplied antibody) and subsequent antigen/antibody complex detection (by agglutination, immunoassay, etc.). A common example is the Cryptococcal antigen test, which routinely relies on agglutination tested at differing titers of CSF. The second means of pathogen antigen detection relies on amplification and detection of nucleic acid sequences from the pathogen of interest. The most common form of this latter detection method is the polymerase chain reaction (PCR) assay. With the advent of more rapid and reliable methods for gene sequencing, more PCR assays are becoming available for use in the detection of even small numbers of pathogens in clinical samples. While many PCR assays are available for use in serum samples (see below), few have been expressly validated for use on CSF samples. While this does not preclude their use in CSF samples, it may limit their clinical utility, particularly in the setting of isolated CNS disease. A list of currently available PCR assays is given in Table 2 .

\section{Cerebrospinal fluid antibody (serologic) studies}

In the absence of antigen detection or in settings where free (i.e. CSF-compartment localized) organism burden may be so low as to preclude detection even with PCR, the detection of intrathecally produced antibody has been the mainstay of diagnosis for many CNS fungal infections. Antibody studies can be attractive for many reasons. First, antibodies to an organism are usually produced in numbers far in excess of the organism itself, thereby leading to easier detection. Second, the antibody molecules are usually much lighter than the organism, leading to greater diffusion in the CSF and a lack of dependence on the vicissitudes of CSF circulation. Third, antibody levels have little dependence on the level of viable organism in the CSF, allowing for empiric treatment of the infection (which can be important in particular CNS infections), with later identification of the etiologic agent. Lastly, antibody production often will spill over into the CSF, even when the infection is isolated to the parenchyma or to the extradural space, making for easier diagnosis of fungal causes of space-occupying lesions and foreign body infections. Unfortunately, antibody studies may not distinguish readily between active infection and prior infection. This can present a problem when the etiologic agent is something to which the person may have previously been exposed. The problem may be even more difficult when the patient is likely infected with a fungal agent, but when it is unclear if a particular CNS clinical presentation is due to that agent or is unrelated to it. Several methods have been devised to better differentiate between prior and active infection and intra- and extra-CNS sources of infection. While these methods have been validated in some instances for bacterial and viral processes, for the most part these are not used routinely in CNS fungal infections.

\section{Blood/plasma studies}

With the exception of foreign body-associated and post-

Table 2: Currently available RT-polymerase chain reaction methods for fung ${ }^{[13]}$

\begin{tabular}{ll}
\hline Genus & Specimens tested \\
Aspergillus & Blood, culture, tissue \\
Candida & Blood, culture oral rinse, tissue \\
Coccidioides & Culture \\
Conidiobolus & Tissue \\
Cryptococcus & Culture, tissue \\
Histoplasma & Culture, tissue \\
Paracoccidioides & Culture \\
Pneumocystis & Oral wash, tissue, sputum \\
Stachybotrys & Culture \\
\hline
\end{tabular}


surgical infections, the CNS almost never represents the primary site of infection, even when the CNS appears to be the sole site affected. In some instances the CNS infection represents disseminated infection, in which case attempts to isolate the causative agent from the peripheral blood may be successful whereas attempts to isolate it from the CSF may not. In other instances, the CNS infection may reflect the sequela of a prior disseminated infection, in which case investigations directed at probing the immune system's response to prior infection may be most fruitful.

\section{Wright's stain}

As is the case with staining methods of the CSF, it is rare that fungal elements will be seen on peripheral smear of blood. There are a few reports of fungemia incidentally diagnosed on peripheral smear, but the implications for diagnosis of fungal infections of the CNS are unclear.

\section{Blood cultures}

Peripheral blood cultures are most likely to be useful when the etiologic agent is a Candida spp. Conversely, given the propensity of Candida spp. to seed distant sites, any finding of candidemia should prompt an evaluation for evidence of CNS infection. It has been estimated that up to $30 \%$ of patients who are candidemic will have evidence of infection metastatic to the retina. This might serve as a useful estimate of rates of other CNS metastasis. Other fungi, such as Histoplasma capsulatum and Cryptococcus neoformans, can occasionally be isolated from blood cultures. Collection of blood specimens in specially formulated "fungal isolator" media may likewise increase the yield of blood cultures for fungal pathogens. ${ }^{[14]}$ While primarily at the case report level, even filamentous molds have been reported to be cultured from peripheral blood in the setting of solid organ manifestations in iatrogenically immunosuppressed patients.

\section{Antigen detection}

As mentioned above, these methods are typically either antibody-based detection of antigen through immune complex formation or PCR-based detection of organism-specific nucleic acid sequences. Also as mentioned above, most of the studies done in CSF are actually developed for use with peripheral blood/serum. For a list of available PCR tests, refer to Table 2.

\section{Serologic studies}

As for CSF, peripheral blood/serum antibodies can help in diagnosis by providing circumstantial evidence for the presence of an etiologic agent in a given host. While subject to the same weaknesses as testing mentioned above for CSF, antibody testing on peripheral blood is often more sensitive as the overall antibody levels are higher in the peripheral blood. Fungal pathogens for which serologic studies are the diagnostic assays of choice include Blastomyces dermatitidis, Coccidioides immitis and Histoplasma capsulatum.

\section{Fungal Pathogens of Interest}

\section{Dimorphic (endemic) fungi}

\section{Histoplasma capsulatum}

Definitive diagnosis is by isolation of the organism, but is accomplished in only a minority of CSF samples from patients with Histoplasma meningitis. Culture yield may be increased to up to $65 \%$, particularly with multiple samples and a larger volume of CSF. ${ }^{[15]}$ Ancillary diagnostic techniques may be used to look for evidence of disseminated Histoplasmosis, which in turn increases the pre-test probability that a particular CNS manifestation is due to Histoplasma infection. Such tests would include antigen detection (ELISA), usually in the urine, serum PCR and acute/convalescent serologies. ${ }^{[16]}$ Such studies routinely have sensitivities of $76-92 \%$, depending on the presenting syndrome and immune status of the host. Unfortunately, PCR has not been validated for the testing of CSF. It is thus often recommended that multiple, large-volume CSF cultures be obtained in conjunction with serum testing to establish the diagnosis of isolated Histoplasma meningitis. ${ }^{[17]}$ In extreme cases, when a diagnosis must be established, cisternal/vetricular sampling and meningeal biopsy have been reported to be useful. ${ }^{[18,19]}$ It is important to remember that specificity of many of the diagnostic methods may be compromised by crossreactivity, particularly with the other dimorphic fungi and Cryptococcus spp..$^{[20,21]}$

\section{Blastomyces dermatitidis}

Like Histoplasma capsulatum, infection with Blastomyces is diagnosed definitively by isolation of the organism. However, it is less often successfully diagnosed than either Histoplasma or Coccidioides, particularly in the setting of isolated CNS infection. ${ }^{[22,23]}$ In the setting of concomitant pulmonary infection, culture of sputum samples may have a diagnostic yield of up to $92 \%$. Wet preparations have had diagnostic yields reported in the $36-46 \%$ range. ${ }^{[24]}$ In some instances, yeast forms have been seen on cytologic examination of the CSF. Serologic methods are occasionally useful, with the most sensitive and specific test being the immunodiffusion test. ${ }^{[25]}$ However, in the setting of isolated anatomic site disease, sensitivity may be as low as $33 \%$. Both nucleic acid and other antigen detection methods are available, though these are not well-studied in CNS infection and as with other mentioned tests, are rarely helpful in isolated disease. Overall, examination of the CSF is rarely helpful in the diagnosis of CNS blastomycosis. ${ }^{[26]}$ In the absence of other positive or suggestive data, biopsy is the diagnostic study of choice. ${ }^{[22]}$

Coccidioides immitis

Similar to the other two dimorphic fungi discussed above, definitive diagnosis rests on isolation of the organism. ${ }^{[27]}$ For isolated CNS infection with Coccidioides, diagnosis can be particularly challenging 
and will often rely on serologic tests. ${ }^{[28,29]}$ The two most commonly used serologic tests are the detection of complement-fixing antibodies and the immunodiffusion tests. Though both latex agglutination tests and ELISAs are available, their utility in the diagnosis of CNS infections has not been established and are therefore discouraged.$^{[28]}$ There are currently no standardized CSF antigen detection methods.

\section{Cryptococcus neoformans}

As infection with this pathogen typically occurs in the setting of immunosuppression, it often also occurs with a relatively high organism burden. This makes its detection much easier than other yeasts. Microscopic examination of CSF using India ink is diagnostic in up to $80 \%$ of AIDS patients. ${ }^{[30]}$ This diagnostic yield can be reproduced (and improved marginally) with the use of Calcofluor white or other direct organism detection methods. ${ }^{[31,32]}$ Of note, Cryptococcal meningitis may occur in non-immunosuppressed hosts, in which case the organism burden is typically much lower and most attempts at staining for the organism have relatively low diagnostic yield. The yeast itself may also be cultured, which may be helpful in establishing the particular subspecies of Cryptococcus involved. Cultures alone are not typically used for diagnosis. The mainstay of the diagnostic methods for Cryptococcus is the polysaccharide antigen serologic assay. There are both latex agglutination and enzyme immunoassay versions in routine use and both are more than $90 \%$ sensitive and specific. ${ }^{[33,34]}$ The tests have been used successfully to detect presence of Cryptococcal antigen before cultures of CSF have turned positive. A PCR test has been reported, ${ }^{[35]}$ but is not yet in mainstream use.

Aspergillus spp.

Unlike the situation with other fungal pathogens, Aspergillus species are ubiquitous and are known to colonize human tissues without necessarily demonstrating invasiveness or pathogenicity. Therefore, a positive serology or even a positive culture, does not necessarily prove pathogenicity. The situation in the CNS is somewhat simplified, as tissues with positive cultures by definition demonstrate invasiveness and pathogenicity. Blood cultures, even in the setting of widespread disseminated disease, are rarely positive. ${ }^{[36]}$ Another complication is that, as Aspergillus infections typically occur in the setting of profound and prolonged immunosuppression, invasive diagnostic methods are significantly limited and are in some cases deemed of greater risk than the initiation of empiric antifungal therapy for a given clinical syndrome consistent with fungal infection. Of the available serologic assays directed against Aspergillus antigens, two are most commonly used. ${ }^{[37]}$ The first is the galactomannan assay, which is an Aspergillus-specific antigen. The second is the (1,3)-beta-D-glucan assay, which is a relatively nonspecific fungal assay. Both tests have been run successfully on CSF samples, ${ }^{[38]}$ though there seems to be more collective clinical experience with use of the galactomannan assay in non-plasma samples. ${ }^{[39,40]}$ In plasma samples, the galactomannan assay has been reported to have a $>95 \%$ sensitivity and specificity. The beta glucan assay has a reported sensitivity of $87 \%$ and a markedly lower specificity, owing not only to its cross-reactivity to a wide range of fungi, but to other glucan-containing products. ${ }^{[41]}$ Recently, a DNA PCR assay for Aspergillus has been reported, with sensitivities approaching $100 \%$ in serum or BAL samples. There is no standardized PCR for Aspergillus in CSF as of this writing, though there are reports of its utility in CSF samples. ${ }^{[42-45]}$

\section{Candida spp.}

Candidal CNS infections typically occur in two clinical scenarios. ${ }^{[46,47]}$ The first is as an encephalitis, occasionally occurring with a meningitis, most often encountered in the setting of disseminated candidiasis. This situation typically involves microabscesses of the brain parenchyma and typically other organs are similarly affected (e.g. liver, spleen). In this situation, staining techniques have a sensitivity of up to $40 \%$ and the organism can be readily cultured from CSF or brain tissues. The second clinical scenario is that of a primary meningitis, usually complicating a foreign body (e.g. ventricular shunt) or other CNS invasive procedure (e.g. surgery, lumbar puncture).$^{[48,49]}$ These infections may have a widely variable course, from rapidly fatal (without treatment) to smoldering, chronic infections. The rapidity of progression typically reflects organism burden, which in turn affects how easily the organism is retrieved from clinical specimens. Of note, Candida infection of ventricular shunt devices may present with widely variable symptoms and, given the low organism burden typically encountered in this setting, may be particularly difficult to diagnose. Particularly in symptomatic cases, the workup may require multiple lumbar punctures and/or ventricular shunt samplings and may even necessitate removal of hardware for adequate diagnosis. As mentioned above, the $(1,3)$-betaD-glucan assay may be useful in diagnosis of Candidal infections of the CNS. ${ }^{[50]}$

\section{Future Trends}

It is clear from the above discussion that the diagnostic methods for CNS fungal infections have lagged behind those for other infective agents. The diagnostic advancements seen for viral pathogens have yet to come to fruition for many fungal pathogens of interest. In part this is due to the relative rarity of fungal infections of the CNS and in part it is due to the lack of a gold standard against which to calibrate or verify studies of new tests. Despite these difficulties, it is anticipated that there will be further evolution of molecular diagnostic methods, 
particularly PCR, leading to the more rapid and more sensitive detection of fungal pathogens in the CNS. Currently, advancements in the field of pharmacology are making treatment of fungal infections of the CNS more palatable and efficacious. It is hoped that the advent of new diagnostic methods will not only lead to a better estimate of the incidence and prevalence of fungal CNS disease in specific populations, but will also permit better trials for the newer available therapeutics, leading to overall better outcomes for our patients.

\section{References}

1. Norlinah MI, Ngow HA, Hamidon BB. Angioinvasive cerebral aspergillosis presenting as acute ischaemic stroke in a patient with diabetes mellitus. Singapore Med J 2007;48:e1-4.

2. Coplin WM, Cochran MS, Levine SR, Crawford SW. Stroke after bone marrow transplantation: Frequency, aetiology and outcome. Brain 2001;124:1043-51.

3. Murthy JM, Sundaram C, Prasad VS, Purohit AK, Rammurti S, Laxmi V. Aspergillosis of central nervous system: A study of 21 patients seen in a university hospital in south India. J Assoc Physicians India 2000;48:677-81.

4. Talmi YP, Goldschmied-Reouven A, Bakon M, Barshack I, Wolf M, Horowitz Z, et al. Rhino-orbital and rhino-orbito-cerebral mucormyeosis. Otolaryngol Head Neck Surg 2002;127:22-31.

5. Radner AB, Witt MD, Edwards JE Jr. Acute invasive rhinocerebral zygomycosis in an otherwise healthy patient: Case report and review. Clin Infect Dis 1995;20:163-6.

6. Siddiqui AA, Shah AA, Bashir SH. Craniocerebral aspergillosis of sinonasal origin in immunocompetent patients: Clinical spectrum and outcome in 25 cases. Neurosurgery 2004;55:602-13.

7. Thomson RB, Bertram H. Laboratory diagnosis of central nervous system infections. Infect Dis Clin North Am 2001;15:1047-71.

8. Zunt JR, Marra CM. Cerebrospinal fluid testing for the diagnosis of central nervous system infection. Neurol Clin 1999;17:675-89.

9. Treseler CB, Sugar AM. Fungal meningitis. Infect Dis Clin North Am 1990;4:789-808.

10. Lo Re V $3^{\text {rd }}$, Gluckman SJ. Eosinophilic meningitis. Am J Med 2003;114:217-23.

11. Ismail Y, Arsura EL. Eosinophilic meningitis associated with coccidioidomycosis. West J Med 1993;158:300-1.

12. McGinnis MR. Detection of fungi in cerebrospinal fluid. Am J Med $1983 ; 75: 129-38$.

13. Espy MJ, Uhl JR, Sloan LM, Buckwalter SP, Jones MF, Vetter EA, et al. Real-Time PCR in clinical microbiology: Applications for routine laboratory testing. Clin Micro Rev 2006;19:165-256.

14. Roberts GD, Washington JA $2^{\text {nd }}$. Detection of fungi in blood cultures. J Clin Microbiol 1975;1:309-10.

15. Deepe GS Jr. Histoplasma capsulatum. In: Mandell GL, Bennett JE, Dolin R, editors. Principles and practice of infectious diseases. $6^{\text {th }}$ ed. Elsevier: Philadelphia, PA; 2005. p 3012-25.

16. Wheat LJ, Kohler RB, Tewari RP, Garten M, French ML. Significance of Histoplasma antigen in the cerebrospinal fluid of patients with meningitis. Arch Intern Med 1989;149:302-4.

17. Wheat LJ, Musial CE, Jenny-Avital E. Diagnosis and management of central nervous system histoplasmosis. Clin Infect Dis 2005;40:84452.

18. Rivera IV, Curless RG, Indacochea FJ, Scott GB. Chronic progressive CNS histoplasmosis presenting in childhood: Response to fluconazole therapy. Pediatr Neurol 1992;8:151-3.

19. Sullivan AA, Benson SM, Ewart AH, Hogan PG, Whitby RM, Boyle RS. Cerebral histoplasmosis in an Australian patient with systemic lupus erythematosus. Med J Aust 1998;169:201-2.

20. Wheat J, French M, Batteiger B, Kohler R. Cerebrospinal fluid Histoplasma antibodies in central nervous system histoplasmosis. Arch
Intern Med 1985;145:1237-40.

21. Paya CV, Roberts GD, Cockerill FR $3^{\text {rd }}$. Laboratory methods for the diagnosis of disseminated histoplasmosis: Clinical importance of the lysiscentrifugation blood culture technique. Mayo Clin Proc 1987;62:480-5.

22. Friedman JA, Wijdicks EF, Fulgham JR, Wright AJ. Meningoencephalitis due to Blastomyces dermatitidis: Case report and literature review. Mayo Clin Proc 2000;75:403-8.

23. Chowfin A, Tight R, Mitchell S. Recurrent blastomycosis of the central nervous system: Case report and review. Clin Infect Dis 2000;30:96971.

24. Chapman S. Blastomyces dermatitidis. In: Mandell GL, Bennett JE, Dolin R, editors. Principles and practice of infectious diseases. $6^{\text {th }}$ ed. Elsevier: Philadelphia, PA; 2005. p. 3026-39.

25. Soufleris AJ, Klein BS, Courtney BT, Proctor ME, Jones JM. Utility of anti-WI-1 serological testing in the diagnosis of blastomycosis in Wisconsin residents. Clin Infect Dis 1994;19:87-92.

26. Kravitz GR, Davies SF, Eckman MR, Sarosi GA. Chronic blastomyeotic meningitis. Am J Med 1981;71:501-5.

27. Galgiani JN. Coccidioides Species. In: Mandell GL, Bennett JE, Dolin R, editors. Principles and practice of infectious diseases. $6^{\text {th }}$ ed. Elsevier: Philadelphia, PA; 2005. p. 3040-50.

28. Johnson RH, Einstein HE. Coccidioidal meningitis. Clin Infect Dis $2006 ; 42: 103-7$.

29. Standard PG, Kaufman L. Immunological procedure for the rapid and specific identification of Coccidioides immitis cultures. J Clin Microbiol 1977;5:149-53.

30. Perfect J. Cryptococeus neoformans. In: Mandell GL, Bennett JE, Dolin R, editors. Principles and practice of infectious diseases. $6^{\text {th }}$ ed. Elsevier: Philadelphia, PA; 2005. p. 2997-3011.

31. Bauters TG, Swinne D, Stove V, Nelis HJ. Detection of single cells of Cryptococeus neoformans in clinical samples by solid-phase cytometry. J Clin Mierobiol 2003;41:1736-7.

32. Sato Y, Osabe S, Kuno H, Kaji M, Oizumi K. Rapid diagnosis of cryptococcal meningitis by microscopic examination of centrifuged cerebrospinal fluid sediment. J Neurol Sci 1999;164:72-5.

33. Kontoyiannis DP. What is the significance of an isolated positive cryptococcal antigen in the cerebrospinal fluid of cancer patients? Mycoses 2003;46:161-3.

34. Asawavichienjinda T, Sitthi-Amorn C, Tanyanont V. Serum cyrptococcal antigen: Diagnostic value in the diagnosis of AIDS-related cryptococeal meningitis. J Med Assoc Thai 1999;82:65-71.

35. Iyer RS, Banker DD. Cryptococcal meningitis in AIDS. Indian J Med Sci 2002;56:593-7.

36. Zarabi MC, Salmassi S. Antemortem diagnosis of systemic aspergillosis: Ten-year review and report of a case. South Med J 1984;77:584-8.

37. Patterson TF. Aspergillus Species. In: Mandell GL, Bennett JE, Dolin R, editors. Principles and practice of infectious diseases. $6^{\text {th }}$ ed. Elsevier: Philadelphia, PA; 2005. p. 2958-72.

38. Viscoli C, Machetti M, Gazzola P, De Maria A, Paola D, Van Lint MT, et al. Aspergillus galactomannan antigen in the cerebrospinal fluid of bone marrow transplant recipients with probable cerebral aspergillosis. J Clin Microbiol 2002;40:1496-9.

39. Machetti M, Zotti M, Veroni L, Mordini N, Van Lint MT, Bacigalupo A, et al. Antigen detection in the diagnosis and management of a patient with probable cerebral aspergillosis treated with voriconazole. Transpl Infect Dis 2000;2:140-4.

40. Verweij PE, Dompeling EC, Donnelly JP, Schattenberg AV, Meis JF. Serial monitoring of Aspergillus antigen in the early diagnosis of invasive aspergillosis. Preliminary investigations with two examples. Infection 1997;25:86-9.

41. Khan ZU, Ahmad S, Mokaddas E, Said T, Nair MP, Halim MA, et al. Cerebral aspergillosis diagnosed by detection of Aspergi llus flavus-specific DNA, galactomannan and (1-->3)-beta-D-glucan in clinical specimens. J Med Microbiol 2007;56:129-32.

42. Hummel M, Spiess B, Kentouche K, Niggemann S, Böhm C, Reuter S, et al. Detection of Aspergillus DNA in cerebrospinal fluid from patients with cerebral aspergillosis by a nested PCR assay. J Clin Microbiol 2006;44:3989-93.

43. Verweij PE, Brinkman K, Kremer HP, Kullberg BJ, Meis JF. Aspergillus meningitis: Diagnosis by non-culture-based microbiological methods and 
management. J Clin Microbiol 1999;37:1186-9.

44. Kami M, Shirouzu I, Mitani K, Ogawa S, Matsumura T, Kanda Y, et al. Early diagnosis of central nervous system aspergillosis with combination use of cerebral diffusion-weighted echo-planar magnetic resonance image and polymerase chain reaction of cerebrospinal fluid. Intern Med 1999;38:45-8.

45. Komatsu H, Fujisawa T, Inui A, Horiuchi K, Hashizume H, Sogo T, et al. Molecular diagnosis of cerebral aspergillosis by sequence analysis with panfungal polymerase chain reaction. J Pediatr Hematol Oncol 2004;26:40-4.

46. Sanchez-Portocarrero J, Perez-Cecilia E, Corral O, Romero-Vivas J, Picazo J.J. The central nervous system and infection by Candida species. Diagn Microbiol Infect Dis 2000;37:169-79.

47. Edwards JE Jr. Candida species. In: Mandell GL, Bennett JE, Dolin R, editors. Principles and practice of infectious diseases. $6^{\text {th }}$ ed. Elsevier: Philadelphia, PA; 2005. p. 2938-57.
48. Nguyen MH, Yu VL. Meningitis caused by Candida species: An emerging problem in neurosurgical patients. Clin Infect Dis 1995;21:323-7.

49. Montero A, Romero J, Vargas JA, Regueiro CA, Sánchez-Aloz G, De Prados F, et al. Candida infection of cerebrospinal fluid shunt devices: Report of two cases and review of the literature. Acta Neurochir (Wien) 2000;142:67-74.

50. Verduyn Lunel FM, Voss A, Kuijper EJ, Gelinck LB, Hoogerbrugge PM, Liem KL, et al. Detection of the Candida antigen mannan in cerebrospinal fluid specimens from patients suspected of having Candida meningitis. J Clin Microbiol 2004;42:867-70.

Accepted on 18-06-2007

Source of Support: Nil, Conflict of Interest: None declared.

\section{Author Help: Reference checking facility}

The manuscript system (ww w.journalonw eb.com) allows the authors to check and verify the accuracy and style of references. The tool checks the references with PubM ed as per a predefined style. Authors are encouraged to use this facility before submitting articles to the journal.

- The style as well as bibliographic elements should be $100 \%$ accurate to get the references verified from the system. A single spelling error or addition of issue number / month of publication will lead to error to verifying the reference.

- Example of a correct style

Sheahan P, O'leary G, Lee G, Fitzgibbon J. Cystic cervical metastases: Incidence and diagnosis using fine needle aspiration biopsy. Otolaryngol Head Neck Surg 2002;127:294-8.

- Only the references from journals indexed in PubM ed w ould be checked.

- Enter each reference in new line, without a serial number.

- Add up to a maximum 15 reference at time.

- If the reference is correct for its bibliographic elements and punctuations, it will be shown as CORRECT and a link to the correct article in PubM ed will be given.

- If any of the bibliographic elements are missing, incorrect or extra (such as issue number), it will be show $n$ as INCORRECT and link to possible articles in PubM ed will be given. 